\title{
Measurement-Based Spatial Correlation and Capacity of Indoor Distributed MIMO System
}

\author{
Yan Zhang, ${ }^{1,2}$ Limin Xiao, ${ }^{2}$ Shidong Zhou, ${ }^{2}$ and Jing Wang ${ }^{2}$ \\ ${ }^{1}$ School of Information and Electronics, Beijing Institute of Technology, Beijing 100081, China \\ ${ }^{2}$ State Key Laboratory on Microwave and Digital Communications, Tsinghua National Laboratory for Information Science and \\ Technology, Department of Electronic Engineering, Tsinghua University, Beijing 100084, China
}

Correspondence should be addressed to Limin Xiao; xiaolm@tsinghua.edu.cn

Received 1 March 2013; Accepted 20 April 2013

Academic Editor: Wenhua Chen

Copyright (c) 2013 Yan Zhang et al. This is an open access article distributed under the Creative Commons Attribution License, which permits unrestricted use, distribution, and reproduction in any medium, provided the original work is properly cited.

\begin{abstract}
Distributed MIMO (D-MIMO) system is one of the candidates for future wireless access networks. In this study, the spatial correlation and capacity in indoor D-MIMO system are presented. All results are from the actual channel measurements in typical indoor scenarios, including office and corridor. Based on measured data, spatial correlation coefficients between distributed transmitting antennas are analyzed. Although the literature about D-MIMO system assumes the small scale fading between distributed antennas is independent, we find that spatial correlation may still exist in specific propagation scenario. This correlation can also degrade the performance of D-MIMO system. To mitigate the impact of spatial correlation, one efficient method is to use transmitting antenna selection technique.
\end{abstract}

\section{Introduction}

Wireless communication systems have achieved fast developments in past years, with mobile data traffic being expected to grow to 10.8 exabytes per month by 2016 [1] and most traffic to occur in indoor environments. Consequently, in the future communication network, indoor wireless access will play a more important role. To realize high throughput and quality in wireless access systems, one potential approach is to use multiple antennas at both sides of the link. Multi-inputmultioutput (MIMO) system is such a technique that can lead significant capacity gain as long as the multipath components of channel are uncorrelated [2]. Most theoretic analyses about MIMO systems are based on this rich scattering assumption. However, it is shown that spatial correlations may exist in real environment for MIMO systems, and these correlations can degrade the system performance obviously [2-6].

In this paper, distributed MIMO (D-MIMO) systems are considered by actual measurements in typical indoor scenarios. Compared with conventional MIMO systems, DMIMO systems are able to provide enough spatial diversity since the antennas are separated [7]. Higher energy efficiency and fairer coverage can also be achieved in D-MIMO systems $[8,9]$. Consequently, D-MIMO architecture is viewed as one potential choice for future wireless access systems.

Primary D-MIMO research work focuses on the theoretical analysis. However, for the application of D-MIMO systems, investigating the real propagation characteristics is essential. Accurate channel models are fundamental for the system design, but there are very few measurement-based analyses [10-12]. In general, it is assumed that there is no spatial correlation between distributed antennas because of large antenna separation and most existing results also verify this assumption.

In this study, we concentrate on the presence of spatial correlation in indoor D-MIMO systems. The relationship between the propagation environment and spatial correlation is considered. All analyses are based on actual channel measurements in typical indoor scenarios. To collect DMIMO channel impulse response (CIR), measurements are conducted in office and corridor scenarios. Through the raw CIR data, the spatial correlation is extracted and then its 


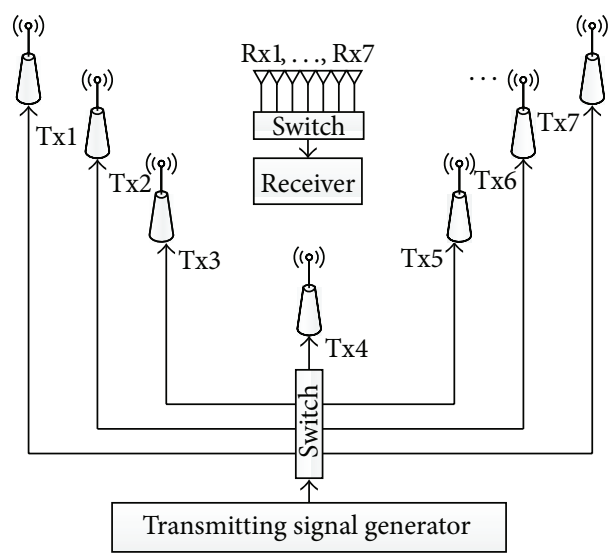

FIgURE 1: D-MIMO measurement system.

TABLE 1: The configurations of THU MIMO channel sounder.

\begin{tabular}{lc}
\hline Carrier frequency & $3.52 \mathrm{GHz}$ \\
Bandwidth & $40 \mathrm{MHz}$ \\
Tx signal length & $12.8 \mu \mathrm{s}$ \\
Snapshot interval & $1.2544 \mathrm{~ms}$ \\
Antenna configuration & $7 \times 7$ \\
Antenna type & Omnidirectional \\
Antenna gain & $4 \mathrm{dBi}$ \\
Polarization & Vertical \\
\hline
\end{tabular}

impact on channel capacity is analyzed. It is shown that in specific scenario there may be high spatial correlation even amongst distributed antennas. And this correlation can also affect the performance of D-MIMO systems. Transmitting antenna selection (TAS) technique is proved to be helpful for counteracting the impact of spatial correlation.

The rest of this paper is organized as follows. In Section 2, the channel sounder and measurement setup are introduced. With measured data, the spatial correlation is analyzed in Section 3. In Section 4, the relationship between spatial correlation and capacity is investigated. Finally, our conclusions are presented in Section 5.

\section{Measurement Setup}

2.1. THU Channel Sounder. Tsinghua University (THU) MIMO channel sounder $[12,13]$ was used to collect raw measured data, which supported both centralized and distributed MIMO channel tests. It worked at the $3.52 \mathrm{GHz}$ central frequency with $40 \mathrm{MHz}$ bandwidth. The major configurations of THU sounder were shown in Table 1.

During this measurement campaign, a signal generator was employed by the transmitter (Tx) to output a wideband test sequence periodically. A 7-port microwave switch was used to connect the signal generator with seven distributed antenna ports, each port with only one Tx antenna. These Tx antennas are omnidirectional with vertical polarization and $4 \mathrm{dBi}$ gain. At the receiver $(\mathrm{Rx})$ side, seven antennas constituted a uniform linear array (ULA) with half-wavelength inter element spacing. The centralized $\mathrm{Rx}$ antennas, which have the same type and gain as the Tx antennas, were also connected with a 7-way switch to the RF tunnel.

As shown in Figure 1, the Tx antennas were distributed while the Rx antennas were centralized. With the switches in both sides, one D-MIMO system was realized by adopting fast time-division-multiplexed switching scheme. These switches were controlled by a synchronization unit and all possible antenna pairs were scanned. The test signal length $t_{p}$ was $12.8 \mu \mathrm{s}$. A guard interval $t_{p}$ was also inserted between adjacent transmissions to protect the test signal from the delay spread infection. The antenna pairs were switched in that fast speed to keep the environment quasi-simultaneous. Then one total snapshot interval was $7 \times 7 \times 2 t_{p}=1254.4 \mu \mathrm{s}$.

The real-time received data was stored in a server. Then the raw data was processed offline to extract the interested channel parameters.

2.2. Measurement Scenarios. The measurements were taken in two typical indoor scenarios (office and corridor) in FIT building, Tsinghua University.

The first scenario was a typical office. The size of the office was $15.6 \mathrm{~m}$ (length) $\times 11.9 \mathrm{~m}($ width $) \times 2.7 \mathrm{~m}$ (height). Typical furniture in this office included wooden desks, plastic chairs, metal cabinets, and computers. As illustrated in Figure 2, seven Tx antennas (blue square markers) were distributed at different locations in the office by using long cables. Tx antenna heights were $1.2 \mathrm{~m}$. The $\mathrm{Rx}$ antenna array was put on a trolley with $1.08 \mathrm{~m}$ height. Then the trolley was moved to different positions. During the campaign, four routes including total 48 positions (red dots) were measured. The length of each route was $13.2 \mathrm{~m}$ and the distance between adjacent positions was $1.2 \mathrm{~m}$. In the following analysis, we would mainly consider two positions in the corner and middle of the office.

The corridor scenario was also selected in FIT building. The corridor length was $105.5 \mathrm{~m}$ and its floor plan was shown in Figure 3. The width and height of the corridor were $2.4 \mathrm{~m}$ and $2.5 \mathrm{~m}$, respectively. There was no furniture in this corridor. Shown as blue square markers, Tx antennas with $7.22 \mathrm{~m}$ separation were distributed in a line along the corridor. During the measurements, the receiver array's position was changed along a designed route, as shown in Figure 3. Total 46 positions with $2 \mathrm{~m}$ separation were selected for data collection. Two positions (red dots) would be taken into account. One was at the end of corridor while the other was in the middle.

\section{Spatial Correlation Analysis}

3.1. Transmitting Spatial Correlation. In D-MIMO system, each base station has $n_{T}$ distributed antenna ports, each port with $l$ microdiversity antennas. The mobile station's antenna number is $n_{R}$ and then this D-MIMO system can be noted by $\left(n_{T}, l, n_{R}\right)[10]$. As mentioned above, in our measurement 


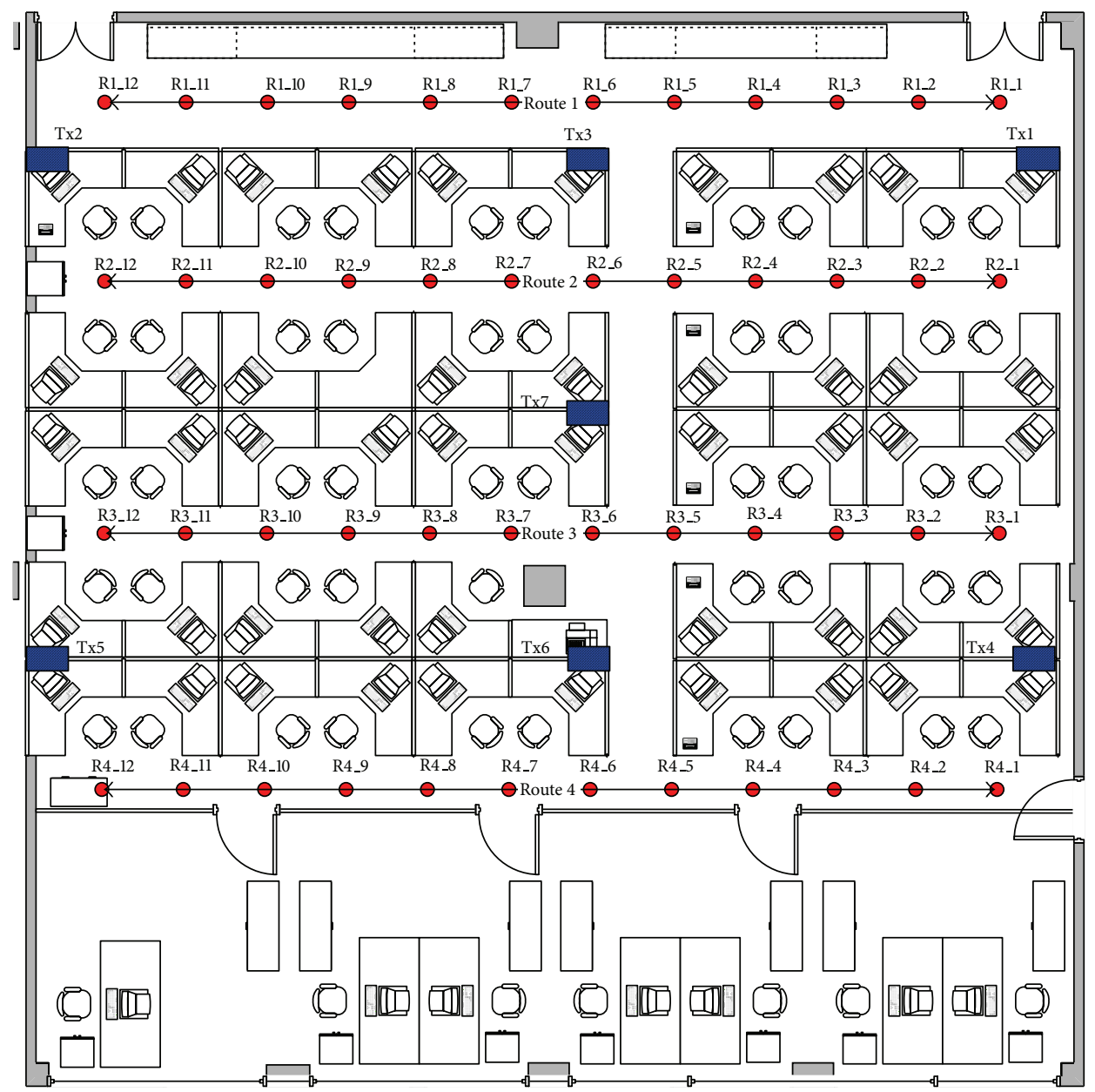

FIgURE 2: Floor plan of the measurement area in office scenario.

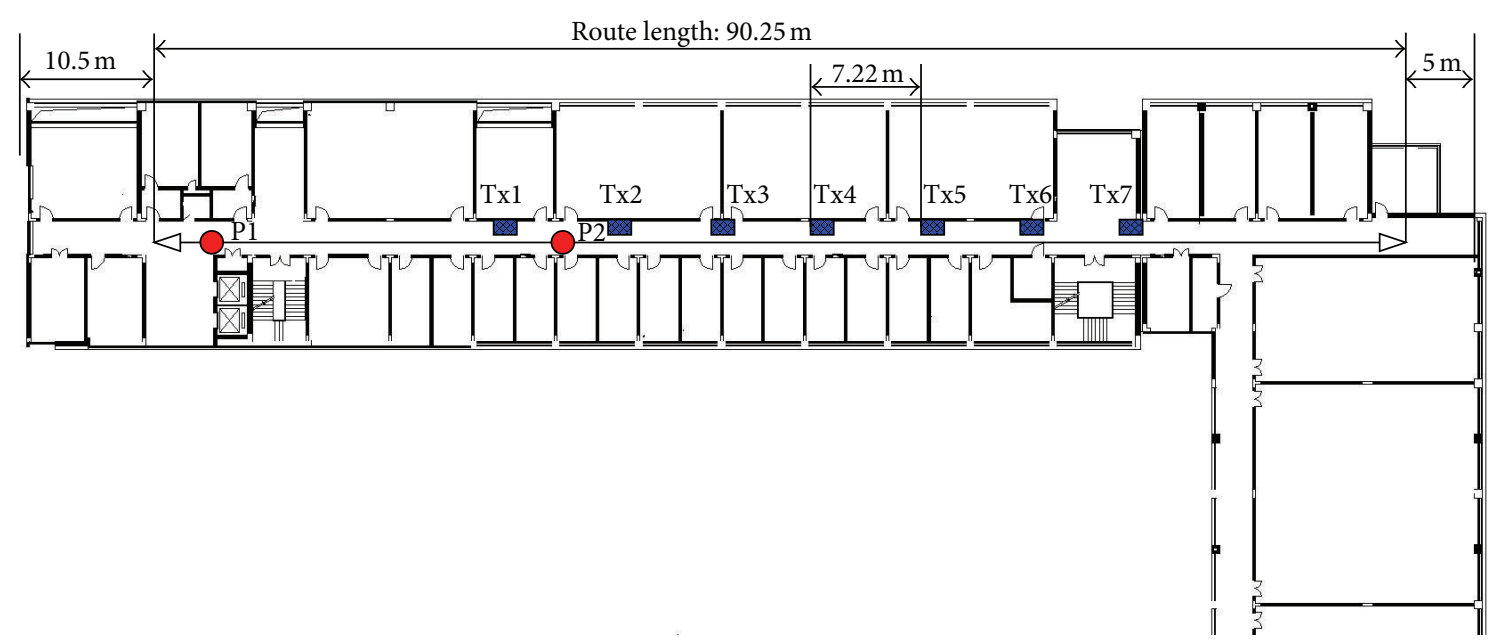

FIGURE 3: Floor plan of the measurement area in corridor scenario.

system $n_{T}=n_{R}=7$ while $l=1$. Then our channel sounder is a $(7,1,7)$ star-shaped D-MIMO system.

From raw measured data, the channel impulse response matrix $\mathbf{H}$ can be extracted. Here $\mathbf{H}$ is an $n_{T} \times n_{R}$ matrix whose elements are the responses between different Tx-Rx antenna pairs.
Due to the centralized placement of $\mathrm{Rx}$ antennas, the correlation at $\mathrm{Rx}$ side in D-MIMO system is similar to that in conventional MIMO systems, which has been well investigated. However, there are still very few considerations about the correlation between distributed Tx antennas. In general, these distributed antennas are assumed independent 
because of large separation $[10,11]$. In this study, we will mainly focus on the Tx spatial correlations and discuss if this assumption is reasonable.

The spatial correlation at Tx side can be defined in the matrix form

$$
\mathbf{R}_{\mathrm{Tx}}=\left(\begin{array}{ccc}
\rho_{11}^{\mathrm{Tx}} & \cdots & \rho_{1 n_{T}}^{\mathrm{Tx}} \\
\vdots & \ddots & \vdots \\
\rho_{n_{T} 1}^{\mathrm{Tx}} & \cdots & \rho_{n_{T} n_{T}}^{\mathrm{Tx}}
\end{array}\right),
$$

which is called the transmitting correlation matrix.

Here $\rho_{i j}^{\mathrm{Tx}}$ is the correlation coefficient between a pair of channels from the $i$ th and $j$ th $\mathrm{Tx}$ antennas, both arriving to the $m$ th Rx element, which is defined as follows [3]:

$$
\rho_{i j}^{\mathrm{Tx}}=\frac{E\left[h_{m i} h_{m j}{ }^{*}\right]-E\left[h_{m i}\right] E\left[h_{m j}{ }^{*}\right]}{\sqrt{\left(E\left[\left|h_{m i}\right|^{2}\right]-\left|E\left[h_{m i}\right]\right|^{2}\right)\left(E\left[\left|h_{m j}\right|^{2}\right]-\left|E\left[h_{m j}\right]\right|^{2}\right)}},
$$

where the superscript ${ }^{*}$ denote conjugation. The $E[\cdot]$ means the average operation of independent samples. The quality of statistical estimates for $\rho_{i j}^{\mathrm{Tx}}$ depends on the number of measured statistical realizations - the more the better [14]. During our measurement, the receiver antenna is moved back and forth in a $10 \lambda$ region at each position. In postprocessing, CIR samples at this region are considered as spatial realizations. The large scale parameter can be viewed as constant in this small region [13]. Consequently, only the small scale correlations among distributed $\mathrm{Tx}$ antennas are involved. Besides spatial realizations, all the frequencies in $40 \mathrm{MHz}$ are used for narrowband analysis. According to (2), $R_{\mathrm{Tx}}$ is a Hermitian matrix.

Based on the measured D-MIMO channel data, we analyze the Tx spatial correlations in the mentioned two scenarios. Firstly, two receiver positions (in Figure 2) in the office scenario are considered. One is position R1_2 in the corner and the other one is position R3_6 in the middle. Figure 4 shows the element magnitudes of transmitting correlation matrices at these two positions. The diagonal elements (autocorrelation coefficients) equal 1, while other elements (cross-correlation coefficients) are near to zero; that is, whether the receiver is placed in the middle or corner, the transmitting spatial correlations are very week in this scenario. Consequently the transmitting correlation matrices $\mathbf{R}_{\mathrm{Tx}}$ can be viewed as an identity matrix, which coincides with the classic assumption.

Then the transmitting spatial correlations in the corridor scenario are calculated and two receiver positions are selected, too. As shown in Figure 3, position P1 is at the end of the corridor while position $\mathrm{P} 2$ is in the middle of Tx1 and Tx2. Figure 5 illustrates the magnitudes of transmitting correlation coefficients at these two positions. When receiver is placed at position $\mathrm{P} 1$, strong spatial correlations can be observed, with correlation coefficients between all Tx antenna pairs being larger than 0.5. That is because signals from different Tx antennas undergo almost the same propagation. Similar reflections and scatterings lead to strong spatial correlations.

As shown in Figure 5(b), if the receiver locates at the position $\mathrm{P} 2$, Tx1 is uncorrelated with other antennas because they are fixed at different sides of the receiver. Signal from Tx1 suffers from different scattering surroundings compared with those sent by other antennas. Those Tx antennas far from the receiver, for example, Tx6 and Tx7, are strongly correlated, which is also led by the similar propagation.

These interesting results in corridor are not fully in agreement with the classic assumption. Even for D-MIMO system, strong spatial correlations may still exist in specific indoor environment; that is, the separation between distributed antennas may not lead to absolute independence. Also the propagation environment itself is an important issue. The high correlations in Figure 5 are caused by the long and narrow corridor structure.

For comparison, we compute the entire cross-correlation coefficients $\left\{\rho_{i j}^{\mathrm{Tx}} \mid i<j\right\}$ at each position, and then gather these coefficients for both scenarios. Figure 6 illustrates the cumulated distribution functions (CDF) of transmitting correlation coefficients. In office scenario, the correlation coefficients are almost less than 0.5, which means that distributed $\mathrm{Tx}$ antennas are uncorrelated in most cases. In this scenario, there are many scatterers including wall, desks, chairs, cabinets, and computers. Rich scattering condition means that signal suffers complex propagation environment. Consequently, the spatial correlations between different $\mathrm{Tx}$ antennas are quite weak.

Meanwhile, the spatial correlations in the corridor scenario are much stronger. In some specific positions, the crosscorrelation coefficients are larger than 0.8. As mentioned above, the scattering environment is simple in this long and narrow corridor. Moreover, Tx antennas are placed in a line. The signals from $\mathrm{Tx}$ antennas travel in similar reflection environment. Then there are strong correlations between different Tx antennas, especially when these antennas are far from the receiver. These spatial correlations can degrade the D-MIMO system performance, which will be discussed in the following sections. When designing a D-MIMO system, we should try to avoid the appearance of high spatial correlation. For example, one feasible way is to distribute the Tx antennas at both sides of corridor rather than in a line.

\section{Capacity Results in D-MIMO System}

4.1. Channel Capacity with Spatial Correlation. In this section, the impact of Tx spatial correlation on D-MIMO channel capacity will be analyzed. Consider a channel unknown at transmitter. The D-MIMO channel capacity with equally allocated transmitting power can be computed as [15]

$$
C=\log _{2} \operatorname{det}\left[\mathbf{I}_{n_{R}}+\frac{P}{\sigma^{2} n_{T}} \mathbf{H H}^{\dagger}\right],
$$

where $\mathbf{I}_{n_{R}}$ is $n_{R} \times n_{R}$ identity matrix. $P$ is the total transmitting power and $\sigma^{2}$ is the noise power. $P / \sigma^{2}$ stands for the transmitting signal-to-noise ratio (SNR). Superscript ${ }^{\dagger}$ denotes the conjugate transposition. 


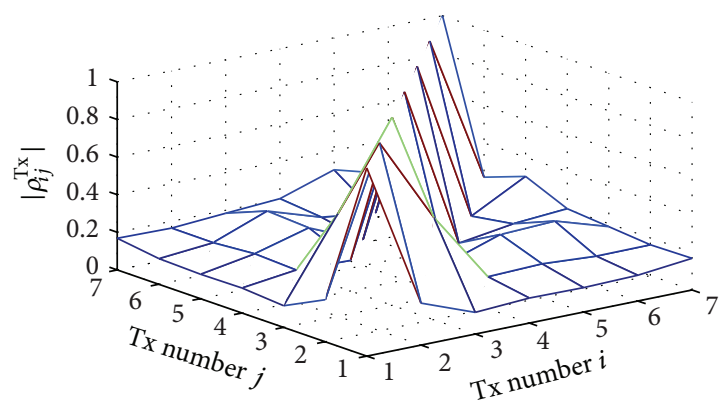

(a)

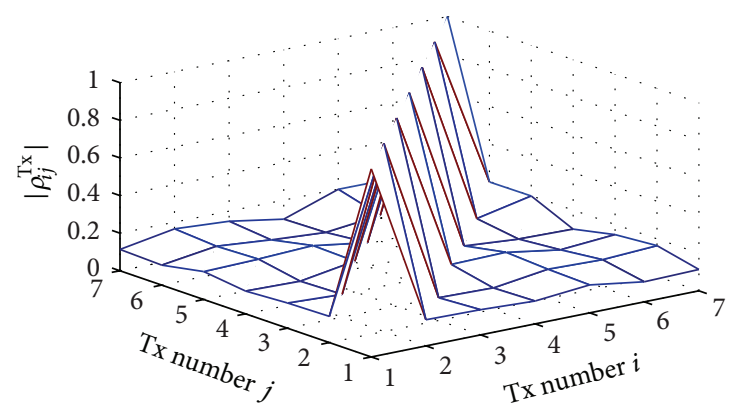

(b)

FIGURE 4: Transmitting correlation coefficients at (a) position R1_2 and (b) position R3_6 in office scenario.

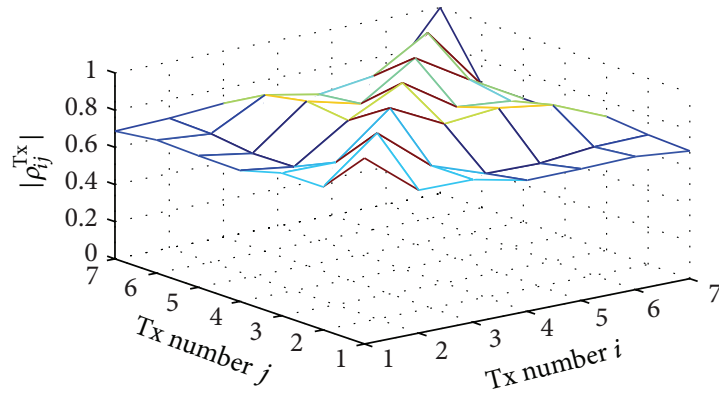

(a)

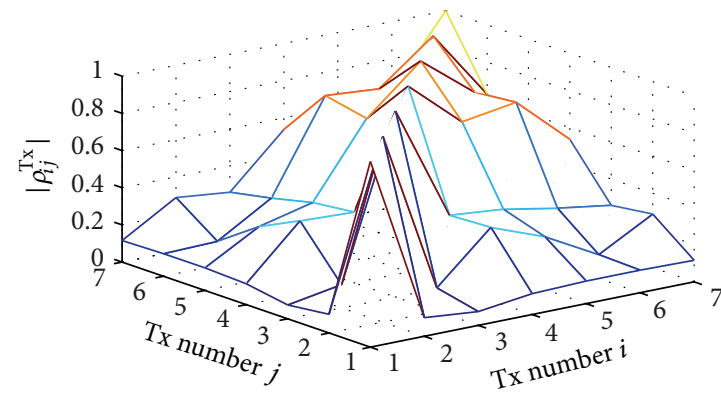

(b)

FIGURE 5: Transmitting spatial correlation coefficients at (a) position P1 and (b) position P2 in corridor scenario.

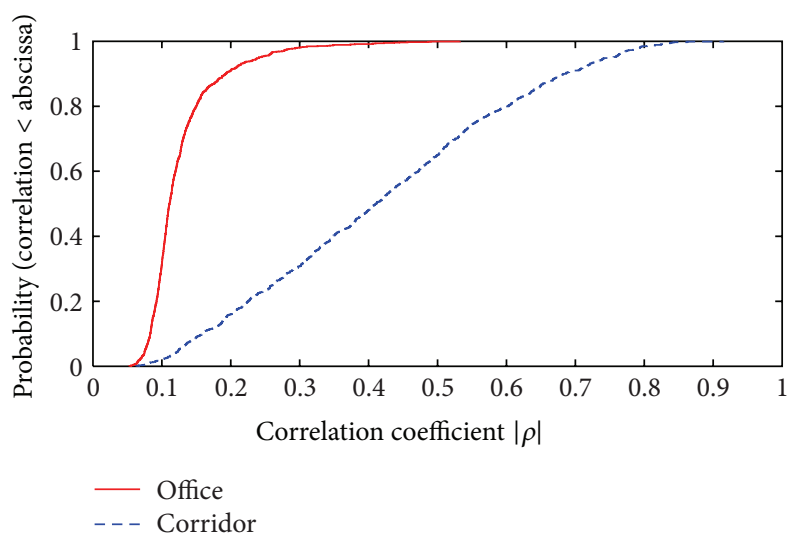

Figure 6: CDF of Tx spatial correlation coefficients for DAS in office and corridor scenarios.

The transmitting correlation matrix $\mathbf{R}_{\mathrm{Tx}}$ is important for the D-MIMO system performance. The existence of spatial correlation can lead the unbalance between eigenvalues of $\mathrm{R}_{\mathrm{Tx}}$ and degrade the MIMO channel capacity [16], which is also proved by our measurement results.

For the selected positions in two scenarios, Figure 7 illustrates the eigenvalue distributions of transmitting correlation matrix. The eigenvalues of $\mathbf{R}_{\mathrm{Tx}}$ satisfy restriction:

$$
\sum_{i=1}^{n_{T}} \lambda_{i}\left(\mathbf{R}_{\mathrm{Tx}}\right)=n_{T} .
$$

Due to the small spatial correlation, in office scenario seven eigenvalues of Tx correlation matrix are very close. In corridor scenario, strong spatial correlation leads the unbalances between eigenvalues. Especially when the receiver is placed at the end of corridor, there are large gaps between the max and the min eigenvalues. As a result, the channel capacity at the corridor end is affected. For example, the measured capacity at position $\mathrm{P} 1$ is $20.8 \mathrm{bit} / \mathrm{s} / \mathrm{Hz}$, which is much smaller than $27.3 \mathrm{bit} / \mathrm{s} / \mathrm{Hz}$ at position $\mathrm{P} 2$.

For an overall analysis, the capacity results in both scenarios are calculated. Figure 8 shows the capacity coverage at all routes in the office scenario. It can be observed that the capacity values are quite close at different positions. By using D-MIMO structure we can expect a uniform capacity coverage, which can provide fairness for users at different positions. This result corresponds to the conclusion introduced by primary research $[8,11]$.

Figure 9 illustrates the capacity coverage in corridor. When the receiver is placed in the middle, that is, $20 \mathrm{~m}$ to $60 \mathrm{~m}$ from the starting receiver point, the capacity coverage is uniform and satisfying. In this area, the Tx spatial correlations are relatively small. While the receiver is fixed at both ends, the capacity values are much smaller than those in the middle. It may be caused by two aspects. The first reason is the strong spatial correlations between different $\mathrm{Tx}$ antennas. As mentioned above, these correlations make the eigenvalues distributed uneven and degrade the D-MIMO system performance. Another important issue may be the large access distance when the receiver is at the end. Long 


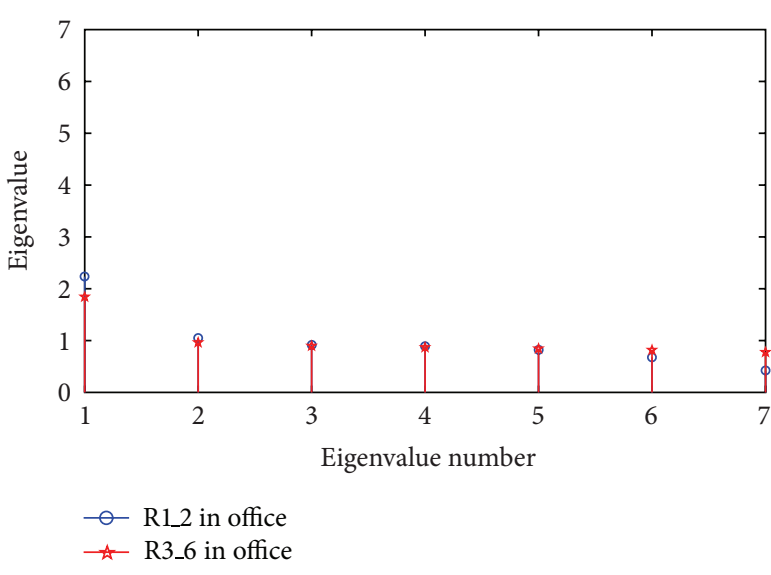

(a)

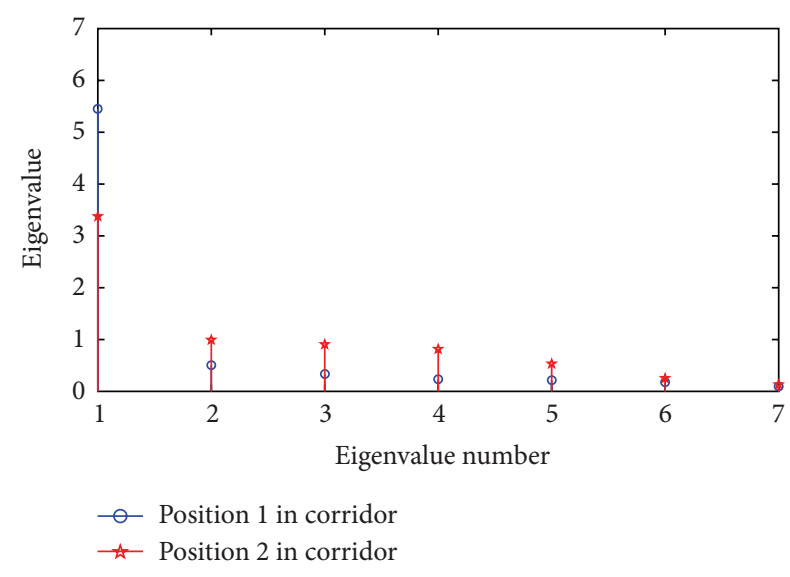

(b)

Figure 7: Eigenvalue distributions at selected positions in (a) office and (b) corridor scenarios.

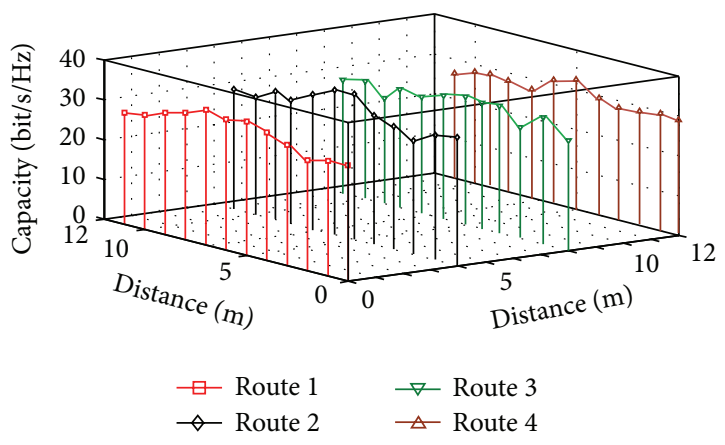

Figure 8: Capacity coverage of D-MIMO system in office scenario.

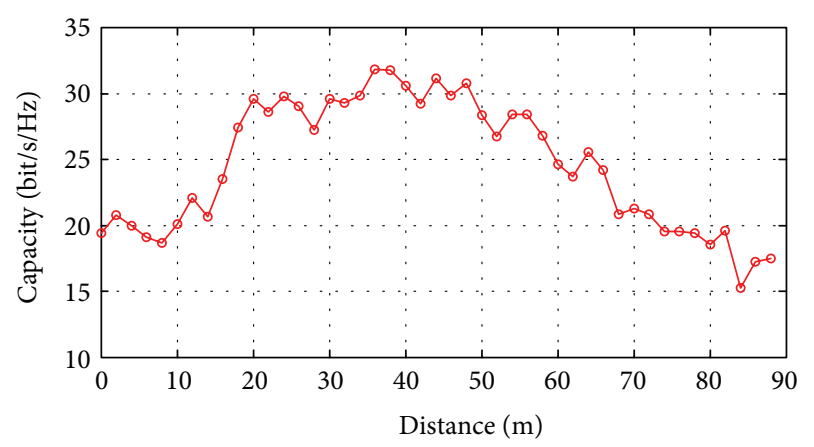

FIGURE 9: Capacity coverage of D-MIMO system in corridor scenario.

access distance brings large pathloss, which weakens received signal strength and degrades the performance.

4.2. Transmitting Antenna Selection in D-MIMO System. Limited to the size and cost requirements, usually mobile terminal only employs three or fewer antennas in practical wireless access systems. Then it is not necessary to use all Tx antennas to serve one user. Especially when Tx antennas are correlated, using more antennas cannot provide more degrees of freedom. It is better to choose fewer Tx antennas with low correlations and high SNR.

TAS technique is able to use the transmitting power efficiently and reduce the processing complexity. At the same time, TAS can lead capacity gain as fixed total transmit power $[17,18]$. In D-MIMO system, the affection of spatial correlation can be partially counteracted, too.

The goal of TAS is to choose an optimum antenna subset to maximize capacity given by [17]

$$
C_{\text {sel }}=\underset{\widetilde{\mathbf{H}} \in \mathbf{H}}{\max }\{C\},
$$

where $\widetilde{\mathbf{H}}$ is a subblock matrix of $\mathbf{H}$.

In the following analysis, only three $\mathrm{Rx}$ antennas $(\mathrm{Rx} 3$, $\mathrm{Rx} 4$, and $\mathrm{Rx} 5$ ) are chosen to simulate a practical terminal. The chosen $\mathrm{Rx}$ array size is one wavelength (about $0.85 \mathrm{~m}$ ). With fixed total transmitting power, the ergodic capacity $\left(C_{\text {all }}\right)$ with all antenna working is computed from the measured data. Then the maximum capacity $\left(C_{\text {opt }}\right)$ with optimum antenna subset is also calculated by searching all possible TAS schemes. The capacity gain as TAS can be defined as

$$
\mathrm{CG}(\%)=\frac{C_{\mathrm{opt}}-C_{\mathrm{all}}}{C_{\mathrm{all}}} .
$$

The TAS capacity gains in corridor scenario are shown in Figure 10. With different number of selected antennas, the total transmitting power is fixed for a fair comparison. Each Tx antenna is assigned equal power and different transmitting SNRs are considered. It is shown that TAS can lead obvious capacity gain, especially under low SNR. Selecting appropriate Tx antenna subset can not only centralize transmitting power but also can weaken the antenna correlation. So TAS technique can be used as a complement of the D-MIMO system, especially in those scenarios with high correlations.

\section{Conclusion}

In this study we analyzed the presence of spatial correlation in indoor D-MIMO system. Field measurements were 


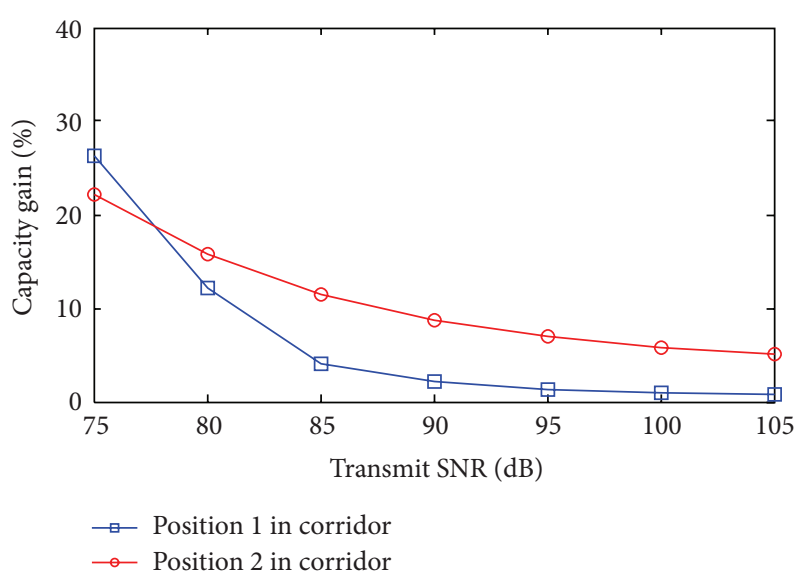

FIGURE 10: TAS capacity gains with different transmitting SNRs in corridor.

conducted in two typical indoor scenarios, including office and corridor. Then the spatial correlations and the capacity results were estimated from measured data. It was proved that in corridor scenario there were spatial correlations between distributed Tx antennas. This correlation could lead the unbalance of eigenvalues and the degradation of channel capacity. To reduce the affection of spatial correlation, transmitting antennas selection technology was able to offer extra capacity gain. These results aimed to provide references for future indoor D-MIMO system design.

\section{Acknowledgments}

This work has been partially supported by National Basic Research Program of China (2013CB329002), National Natural Science Foundation of China (61201192), Tsinghua University Initiative Scientific Research Program (2011Z02292), International S\&T Cooperation Program (2012DFG12010), National S\&T Major Project (2013ZX03001024), Key Grant Project of Chinese Ministry of Education (no. 313005), and China Postdoctoral Science Foundation, China Mobile Research Institute, and Open Research Fund of National Mobile Communications Research Laboratory, Southeast University (2012D02).

\section{References}

[1] Cisco Visual Networking Index: Global Mobile Data Traffic Forecast Update, 2011-2016, White Paper, CISCO Systems Inc., February 2012.

[2] D. S. Shiu, G. J. Foschini, M. J. Gans, and J. M. Kahn, "Fading correlation and its effect on the capacity of multielement antenna systems," IEEE Transactions on Communications, vol. 48, no. 3, pp. 502-513, 2000.

[3] P. L. Kafle, A. Intarapanich, A. B. Sesay, J. McRory, and R. J. Davies, "Spatial correlation and capacity measurements for wideband MIMO channels in indoor office environment," IEEE Transactions on Wireless Communications, vol. 7, no. 5, pp. 15601571, 2008.
[4] P. Almers, F. Tufvesson, and A. F. Molisch, "Keyhole effect in MIMO wireless channels: measurements and theory," IEEE Transactions on Wireless Communications, vol. 5, no. 12, pp. 3596-3604, 2006.

[5] S. Büyükçorak and G. Karabulut Kurt, "Spatial correlation and MIMO capacity at $2.4 \mathrm{GHz}$," in Proceedings of the International Workshop on Modeling and Simulation of Wireless Channels, Meksiko, 2012.

[6] D. P. McNamara, M. A. Beach, and P. N. Fletcher, "Spatial correlation in indoor mimo channels," in Proceedings of the 13th IEEE International Symposium on Personal, Indoor and Mobile Radio Communications, vol. 1, pp. 290-294, September 2002.

[7] A. A. M. Saleh, A. J. Rustako, and R. S. Roman, "Distribute antennas for indoor radio communications," IEEE Transactions on Communications, vol. 35, no. 12, pp. 1245-1251, 1988.

[8] S. Zhou, M. Zhao, X. Xu, J. Wang, and Y. Yao, "Distributed wireless communication system: a new architecture for future public wireless access," IEEE Communications Magazine, vol. 41, no. 3, pp. 108-113, 2003.

[9] C. X. Wang, X. Hong, X. Ge, X. Cheng, G. Zhang, and J. Thompson, "Cooperative MIMO channel models: a survey," IEEE Communications Magazine, vol. 48, no. 2, pp. 80-87, 2010.

[10] M. Alatossava, A. Taparugssanagorn, V. M. Holappa, and J. Ylitalo, "Measurement based capacity of distributed MIMO antenna system in Urban microcellular environment at $5.25 \mathrm{GHz}$," in Proceedings of the IEEE Vehicular Technology Conference (VTC Spring '08), pp. 430-434, Singapore, May 2008.

[11] R. Ibernon-Fernandez, J. M. Molina-Garcia-Pardo, and L. JuanLlacer, "Comparison between measurements and simulations of conventional and distributed MIMO system," IEEE Antennas and Wireless Propagation Letters, vol. 7, pp. 546-549, 2008.

[12] Z. H. Li, F. Y. Luan, Y. Zhang, L. M. Xiao, L. F. Huang, and S. D. Zhou, "Capacity and spatial correlation measurements for wideband distributed MIMO channel in aircraft cabin environment," in Proceedings of the IEEE Wireless Communications and Networking Conference (WCNC '12), pp. 1175-1179, Paris, France, 2012.

[13] Y. Zhang, O. Edfors, and P. Hammarberg, "A general coupling based model framework for wideband MIMO channels," IEEE Transaction on Antennas and Propagation, vol. 60, no. 2, pp. 574-586, 2012.

[14] H. Özcelik, Indoor MIMO channel models [Ph.D. dissertation], Institut für Nachrichtentechnik und Hochfrequenztechnik, TechnischeUniversität, Wien, Austria, December 2004, http://www.nt.tuwien.ac.at/mobile/theses_finished/.

[15] A. F. Molisch, Wireless Communications, IEEE Press-Wiley, Chichester, UK, 2005.

[16] X. Gao, B. Jiang, X. Li, A. B. Gershman, and M. R. McKay, "Statistical eigenmode transmission over jointly correlated MIMO channels," IEEE Transactions on Information Theory, vol. 55, no. 8, pp. 3735-3750, 2009.

[17] S. Sanayei and A. Nosratinia, "Capacity of MIMO channels with antenna selection," IEEE Transactions on Information Theory, vol. 53, no. 11, pp. 4356-4362, 2007.

[18] Y. Zhang, Z. H. Li, and F. Y. Luan, "Measurement-based analysis of transmit antenna selection for in-cabin distributed MIMO system," International Journal of Antenna and Propagation, vol. 2012, Article ID 598049, 6 pages, 2012. 

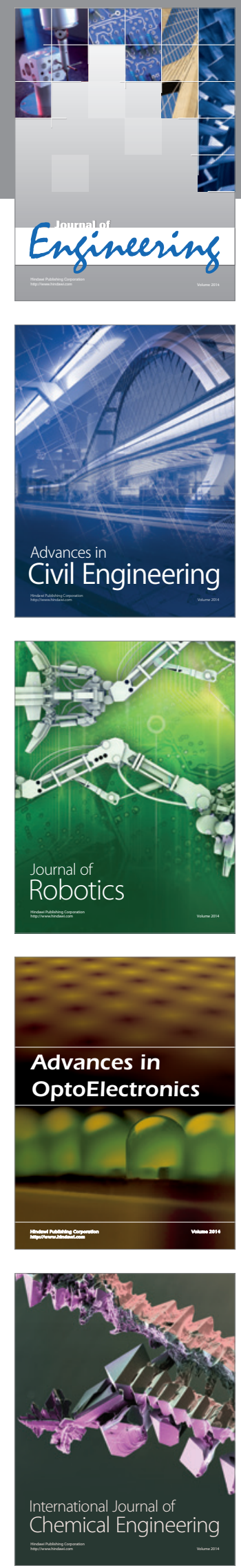

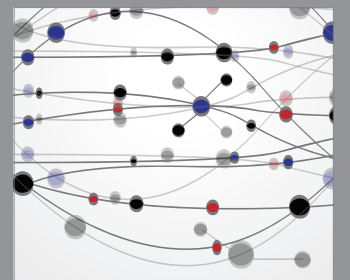

The Scientific World Journal
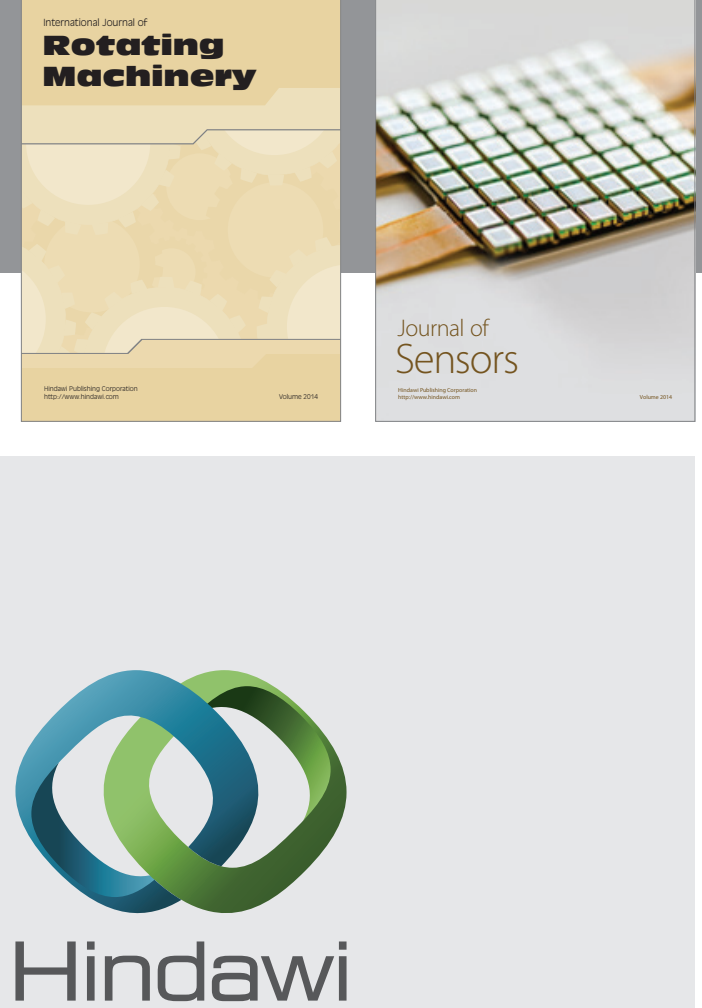

Submit your manuscripts at http://www.hindawi.com
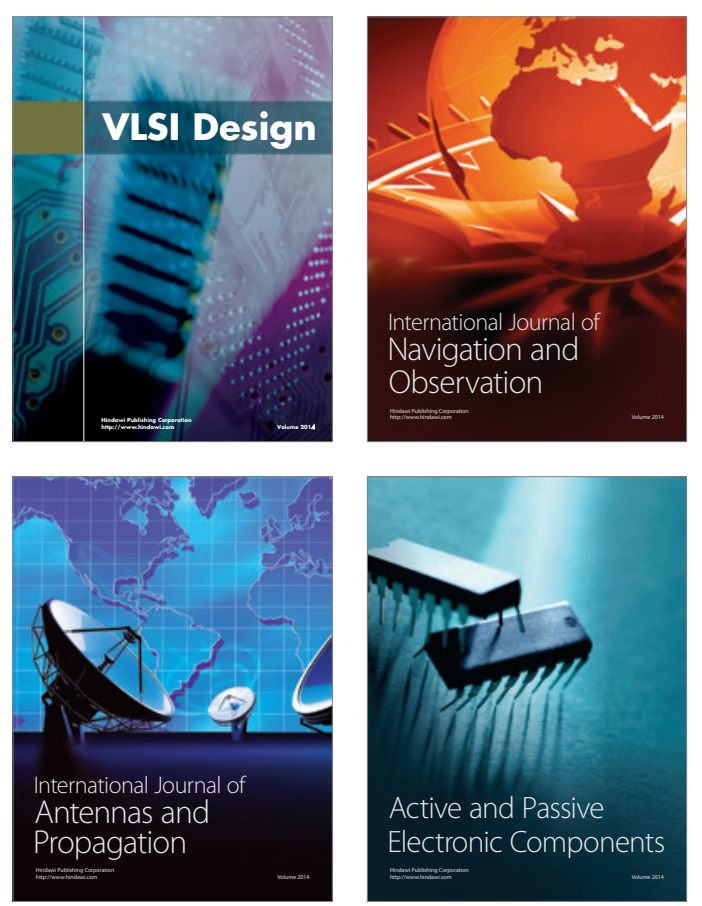
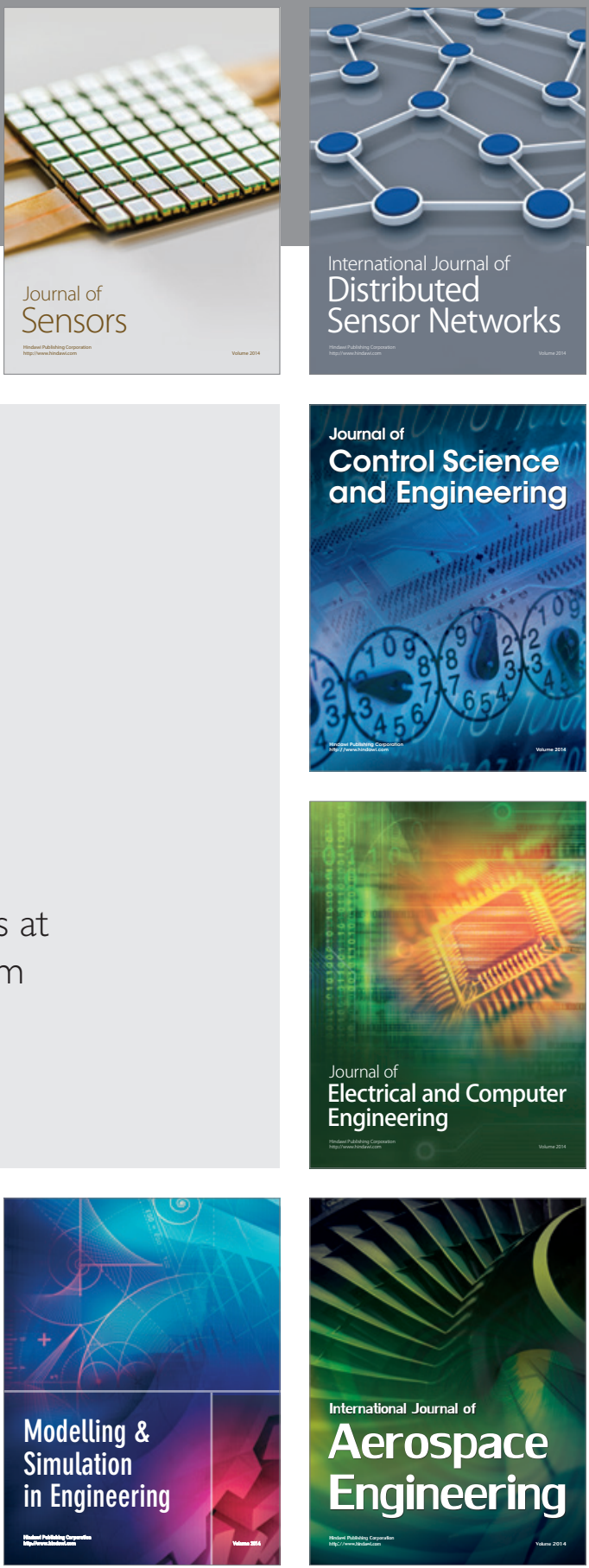

Journal of

Control Science

and Engineering
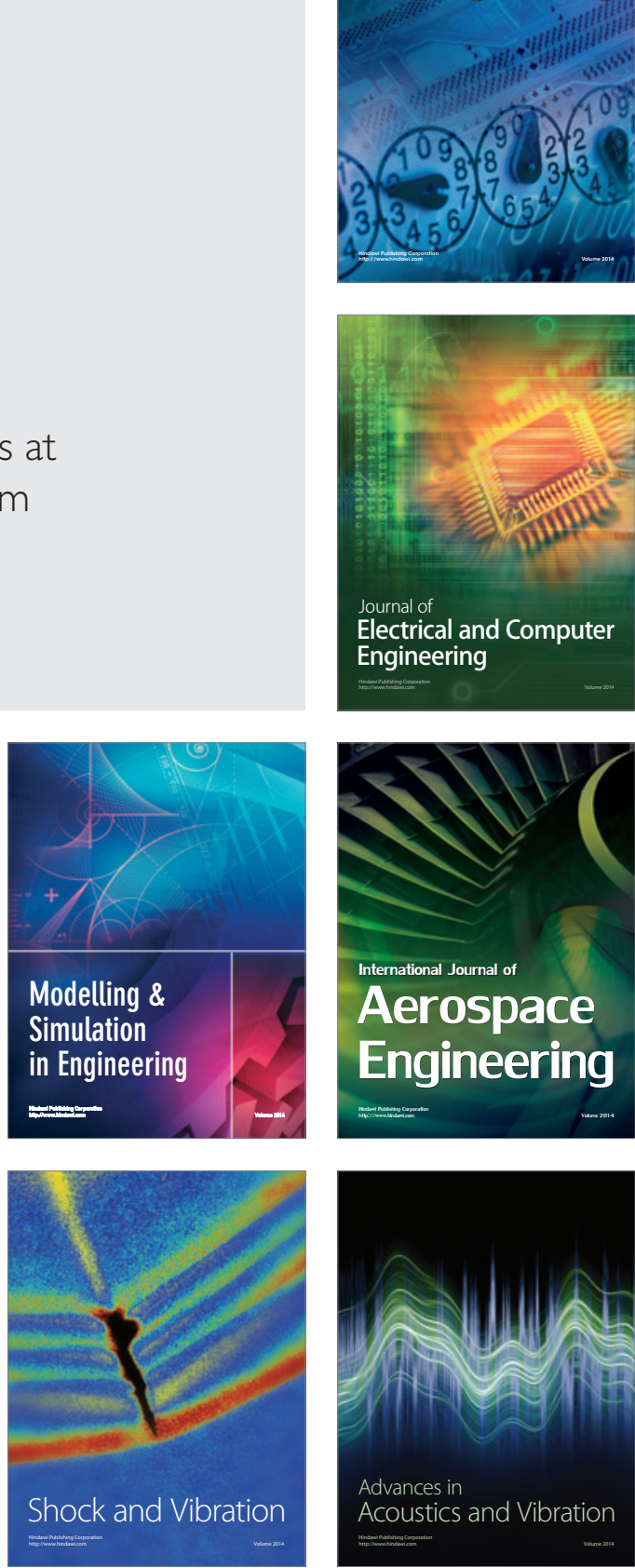